\title{
Sense of meaning as a predictor of burnout in emergency physicians in Israel: a national survey
}

\section{Shulamit Ben-Itzhak ${ }^{1 *}$, Jonathan Dvash ${ }^{1,2 *}$, Maya Maor ${ }^{3,4}$, Noa Rosenberg ${ }^{5,6}$, Pinchas Halpern ${ }^{6,7}$}

'Psychological Service, Tel Aviv Sourasky Medical Center, Tel Aviv, Israel

${ }^{2}$ School of Education, Bar Ilan University, Ramat-Gen, Israel

${ }^{3}$ The Gender Studies Program, Faculty of Humanities and Social Science, Ben-Gurion University of the Negev, Beer-Sheva, Israel

${ }^{4}$ The Women's and Gender Studies Program, Haifa University, Haifa, Israel

${ }^{5}$ Department of Psychiatry, Tel Aviv Sourasky Medical Center, Tel Aviv, Israel

${ }^{6}$ Sackler Faculty of Medicine, Tel Aviv University, Tel Aviv, Israel

${ }^{7}$ Emergency Department, Tel Aviv Souraski Medical Center, Tel Aviv, Israel

Objective Burnout is common in physicians and particularly acute in emergency physicians. Physician burnout may adversely affect physicians' lives and the quality of care they provide, but much remains unknown about its main contributing factors. The present study evaluated burnout rates and contributing factors in emergency physicians in Israel, specifically focusing on the role of a sense of meaning, which has received little attention in the literature concerning burnout in emergency physicians.

Methods A multicenter study, involving a convenience sample of physicians working full-time in the emergency departments of 16 general hospitals in Israel, was conducted. Questionnaires were used to assess burnout, demographic characteristics, professional stress, emotional distress, satisfaction, and quality of professional life, and open-ended questions were used to evaluate subjective perception of job satisfaction.

Results Seventy physicians completed the questionnaires; $71.4 \%$ reported significant burnout levels in at least one of the burnout measures, while $82 \%$ also reported medium or high levels of competency. Burnout levels were associated with work-life balance, work satisfaction, social support, depressive symptoms, stress, and preoccupying thoughts. Regression analysis yielded two significant factors associated with burnout: worry and a sense of existential meaning derived from work. In addition, $61 \%, 51 \%$, and $17 \%$ of participants exhibited high emotional exhaustion, high depersonalization, and a low sense of personal accomplishment, respectively.

Conclusion These results indicate a high burnout rate in emergency physicians in Israel and highlight relevant positive and negative factors including the importance of addressing existential meaning in designing specific intervention programs to counter burnout.

Keywords Burnout, professional; Emergency medicine; Existential meaning
Received: 30 June 2015

Revised: 18 August 2015

Accepted: 20 August 2015

Correspondence to:

Shulamit Ben-Itzhak

Psychological Service, Tel Aviv Sourasky Medical Center, 6 Weizmann Street Tel Aviv 64239, Israel

E-mail: shulamit@tasmc.health.gov.il

*These two authors contributed equally to this work.

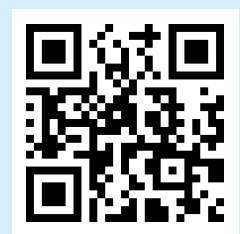

How to cite this article:

Ben-Itzhak S, Dvash J, Maor M, Rosenberg $N$, Halpern P. Sense of meaning as a predictor of burnout in emergency physicians in Israel: a national survey. Clin Exp Emerg Med 2015;2(4):217-225.

This is an Open Access article distributed under the terms of the Creative Commons Attribution Non-Commercial License (http:// creativecommons.org/licenses/by-nc/3.0/). 


Capsule
$\begin{aligned} & \text { What is already known } \\ & \text { The emotional and physical challenges of emergency medicine render burnout a particularly acute problem for this } \\ & \text { population. Sense of meaning is a central factor that may be associated with burnout. }\end{aligned}$
$\begin{aligned} & \text { What is new in the current study } \\ & \text { No studies have examined the significance of existential meaning derived from work as a protective factor against burn- } \\ & \text { out in physicians. Some evidence regarding this relationship was found in studies examining other professionals. In light } \\ & \text { of these findings, the present study examined the impact of existential meaning on burnout in emergency physicians. }\end{aligned}$

\section{INTRODUCTION}

Burnout is a syndrome involving mental and psychological exhaustion, a reduced sense of accomplishment and self-esteem, and depersonalization in individuals whose work involves dealing with people. ${ }^{1-4}$ The syndrome develops as a response to an inability to cope effectively with prolonged stress at work. ${ }^{5}$

While burnout has adverse effects in every occupational setting, it is particularly detrimental to physicians. ${ }^{6,7}$ Burnout in physicians is associated with poor mental and physical health, guilt, poor judgment, drug abuse, decreased satisfaction with work, sleep disturbances, hypertension, posttraumatic stress disorder, anxiety, alcoholism, and myocardial infarction. ${ }^{4,8-11}$ The specific emotional and physical challenges of emergency medicine (EM) render burnout a particularly acute problem for this specific population. ${ }^{11}$

A comprehensive review conducted in 2013 identified only seven studies that examined burnout in emergency physicians (EPs). ${ }^{4}$ To the best of our knowledge, no studies have been conducted to examine burnout in EPs in Israel. Based on the few studies conducted, the following three clusters were found to increase burnout: 1) dissatisfaction with institutional/structural settings (e.g., specialty services); 2) work overload (e.g., an increased number of shifts per month and working hours per week); and 3) emotional issues (e.g., high levels of anxiety due to concern regarding adverse clinical outcomes). ${ }^{7,12,13}$ Factors such as support from others, academic activity, and work/leisure balance potentially increase resilience to burnout. ${ }^{4,14,15}$

Sense of meaning is a central factor that may be associated with burnout. A sense of meaning has long been established as an essential factor in coping with difficult conditions. ${ }^{16}$ Burnout can be characterized as a symptom of an inadequate level of existential meaning manifesting as reduced vitality on both somatic and psychological levels. ${ }^{17}$ Moreover, some reports have indicated that a sense of meaning derived from work was associated with positive outcomes such as increased well-being, enhanced moti- vation, and happiness. ${ }^{18}$ Nevertheless, to the best of our knowledge, no studies have examined the significance of existential meaning derived from work as a protective factor against burnout in physicians. Some evidence regarding this relationship was found in studies examining other professionals (e.g., teachers and managers). ${ }^{19,20}$ In light of these findings, the present study examined the impact of existential meaning on burnout in EPs.

EM is a relatively new specialty in Israel; there are 28 general emergency departments (EDs) in the country. Physician staffing is variable, with regular emergency staff consisting of EM-trained physicians, and full-time non-EM-trained physicians, most of whom are trained in internal medicine or general surgery. Afterhours care is provided mainly by residents in medicine, surgery, and orthopedics, who treat patients in the ED under the supervision of EPs. In total, there are approximately 300 physicians working in each ED on a full- or part-time basis, approximately 150 of whom hold board certification in EM. Residency training is available in two tracks: as a primary or secondary specialty (after board certification in internal medicine, surgery, anesthesiology, family medicine, or pediatrics). There are currently approximately $80 \mathrm{EM}$ residents in training nationally.

The aim of the current study was to explore burnout levels and delineate the contributive and protective factors for burnout in EPs in Israel. This is particularly important in a local context, as a series of reforms and institutional changes implemented in the mid-1990s (associated with budget cuts and a general decline in the social status of doctors) led to an increase in burnout levels in physicians in Israel. ${ }^{21}$

The hypotheses of the present study were as follows: 1) There is a high level of burnout in EP's, mainly due to large workloads. 2) A sense of existential meaning derived from work is a significant factor in countering and coping with burnout. 3) Negative emotions, such as worry or anxiety, increase burnout levels. 4) Factors contributing to well-being, such as work satisfaction and good work-family balance, are significant factors in countering burnout. 


\section{METHODS}

\section{Study design}

This was a multicenter study involving a convenience sample of physicians who worked full-time in the EDs of 16 general hospitals in Israel. Institutional review board approval was obtained from the Tel Aviv Medical Center.

\section{Study setting and population}

Questionnaires were mailed to ED chairpersons, who invited fulltime EPs to complete them. Informed consent was obtained prior to participation. It was impossible to ascertain the exact number of physicians who were invited to participate. As mentioned, the total number of full-time EPs working in EDs in Israel is approximately 300, but approximately 200 work in the 16 participating EDs. Seventy physicians responded (response rate 35\%).

\section{Survey content}

The scale included demographic variables and information regarding training types, work and leisure time, involvement in malpractice suits and mental and physical health including mood and signs of depression and anxiety. The questionnaire included primarily closed-ended questions, with responses provided using the five-point ordinal polytomous Likert scale (with a "not applicable" option), with several open-ended questions and a space for general comments at the end. The specific content of the questionnaire items (originally in Hebrew) is shown in Table 1.

\section{Burnout measurements}

The questionnaire was adapted from a well-established scale, the Maslach Burnout Inventory (MBI). ${ }^{2}$ The $\mathrm{MBI}$ contains 22 items used to assess the three burnout components: emotional exhaustion (9 items), depersonalization (5 items), and personal accomplishment (8 items). Physicians were considered to be experiencing burnout if they exhibited high emotional exhaustion and/or depersonalization scores. ${ }^{22}$ A collective score was generated and compared with group norms published for nurses and physicians (emotional exhaustion, 22.19; personal accomplishment, 36.53; depersonalization, 7.19). ${ }^{2}$

\section{Data analysis}

Standard descriptive summary statistics were used to characterize the physicians. A series of nonparametric Mann-Whitney tests were performed to identify differences between burnout groups (burned out and not burned out) according to the test variables. Significant variables determined via these tests were entered as potential predictor variables in the logistic regression analysis, to identify the variables that were independently predictive of a disposition toward burnout (measured via the MBI).

Further analysis of participants' responses to the open-ended questions was performed to determine the subjective and relevant meanings that respondents attached to their work. Statistical analysis was performed using the IBM SPSS Statistics ver. 21 (IBM Corp., Armonk, NY, USA).

\section{RESULTS}

\section{Demographic characteristics}

Table 2 presents some of the demographic characteristics of physicians who participated in the study; 49, 14, and 7 were specialists, EM residents, and residents in EM as a super specialty (i.e., board certified in another specialty), respectively. 70\% were male, aged 41 to 60 years, married, and had $>25$ years of experience in medicine (for results concerning the other variables included in the survey see Supplementary Table 1).

\section{Burnout}

In total, 61\%, 51\%, and 17\% of respondents exhibited high emotional exhaustion, high depersonalization, and a low sense of personal accomplishment, respectively. In addition, 71.4\% reported experiencing at least one burnout symptom based on a high emotional exhaustion or depersonalization score. Responding physicians' burnout characteristics are summarized in Table 3.

\section{Predictors of burnout}

A series of nonparametric Mann-Whitney tests were performed to determine the differences between burnout groups (burned out and not burned out) according to the test variables. The test variables for which scores differed significantly between groups were as follows: smoking frequency $(P<0.05)$, satisfaction with work $(P<0.001)$, work-life balance $(P<0.05)$, extent to which work provides meaning $(P<0.05)$, social support $(P<0.05)$, feeling depressed $(P<0.001)$, lack of interest in daily activities $(P<0.05)$, preoccupying thoughts $(P<0.05)$, stress $(P<0.001)$, and worry $(P<0.001)$. All correlations were in the expected direction (Table 4).

Logistic regression analysis, including the significant variables outlined above as potential predictor variables, was performed to identify variables that were independently predictive of a disposition toward burnout (measured via the MBI). Two variables predicted burnout: 1$)$ degree of worry $(\beta=3.678, P<0.01$; $95 \%$ confidence interval $[\mathrm{Cl}], 6.28$ to 1.08 ) and 2) extent to which work provides meaning $(\beta=-3.144, \mathrm{P}<0.05 ; 95 \% \mathrm{Cl},-6.24$ to -0.045$)$. 
Table 1. Survey questionnaire

\begin{tabular}{|c|c|}
\hline Variable & Scale \\
\hline Age & $<40,41-45,46-50,51-55,56-60,61-65,>65$ \\
\hline Sex & Male, female \\
\hline Marital status & Single, stable partner, married, separated, divorced, divorced/remarried \\
\hline What is the occupation of your partner? & Open question \\
\hline No. of children & $0,1,2,3,4,5,6,>6$ \\
\hline Do you engage in any physical activity? & Never, rarely, regularly, engage in a lot of physical activity \\
\hline Do you smoke? & Never, rarely, medium degree, heavy smoker \\
\hline Do you drink alcohol? & Never, rarely, medium degree, heavy drinker \\
\hline Do you consume other substances? & Open question \\
\hline Please indicate the setting of your practice & Open question \\
\hline If you practice in a university setting, what is your academic rank? & $\begin{array}{l}\text { Instructor, assistant professor, associate professor, professor, emeritus, not applicable, } \\
\text { other }\end{array}$ \\
\hline How many years have you practiced medicine? & $<5,5-10,11-15,16-20,21-25,>25$ \\
\hline How many years have you practiced emergency medicine? & $<5,5-10,11-15,16-20,21-25,>25$ \\
\hline Have you received other formal specialty training? & No, yes (elaborate) \\
\hline Have you received formal subspecialty training? & No, yes (elaborate) \\
\hline On average, how many clinical hours do you work in the emergency unit per week? & $<30,30-40,41-50,51-60,61-70,71-80,>80$ \\
\hline $\begin{array}{l}\text { On average, how many clinical hours do you work outside the emergency unit per } \\
\text { week? }\end{array}$ & $<30,30-40,41-50,51-60,61-70,71-80,>80$ \\
\hline How many on-call days do you take per month? & $<3,3-5,6-10,>10$ \\
\hline On average, how many hours per week do you spend teaching residents/students? & $0,<5,5-10,11-15,16-20,<20$, irrelevant \\
\hline $\begin{array}{l}\text { On average, how many hours per week do you spend on research (clinical or basic } \\
\text { science)? }\end{array}$ & $<5,5-10,11-15,16-20,>20$ \\
\hline On average, how many hours per week do you spend on administrative tasks? & $<5,5-10,11-15,16-20,>20$ \\
\hline On average, how many weeks of vacation do you take per year? & $<1,1-2,3-4,>4$ \\
\hline Approximately how many minutes is your average daily commute to work & $<10,10-20,21-30,>30$ \\
\hline
\end{tabular}

Over the last 5 years, how many times have you been named in a malpractice lawsuit? None, 1-5, 6-10, > 10

During the past 12 months, have you had suicidal thoughts?

To what degree are you satisfied with your work?

No, yes

Very satisfied, usually satisfied, sometimes satisfied, rarely satisfied, not satisfied at all

Please indicate what gives you satisfaction with your work

Please indicate what makes you unsatisfied about your work

Which change would most significantly improve your job satisfaction?

Do you suffer from any major physical illness?

Do you suffer from any major psychiatric illness?

To what degree do you agree with the statements below?

My work schedule leaves me enough time for my personal/family life

My job gives me meaning

When difficulties arise at work I have someone to talk to about them

How often do you experience the following?

I feel depressed and hopeless

I have little interest in my daily activities

I am under a lot of pressure

I worry a lot

I have thoughts that I cannot get out of my head

I can't stop thinking of work even when I am not there

Open question

Open question

Open question

No, yes (elaborate)

No, yes (elaborate)

Strongly agree, agree, neutral, disagree, strongly disagree

Strongly agree, agree, neutral, disagree, strongly disagree

Strongly agree, agree, neutral, disagree, strongly disagree

Never, few times a year, monthly, few times a month, weekly, few times a week, daily Never, few times a year, monthly, few times a month, weekly, few times a week, daily Never, few times a year, monthly, few times a month, weekly, few times a week, daily Never, few times a year, monthly, few times a month, weekly, few times a week, daily Never, few times a year, monthly, few times a month, weekly, few times a week, daily Never, few times a year, monthly, few times a month, weekly, few times a week, daily

\section{Open-ended questions}

Open-ended questions facilitated the identification of the subjective and relevant meanings respondents attached to their work. First, analysis of the most satisfying elements of respondents' work indicated the dominance of factors that gave existential meaning

to their work. Of the five clusters of responses identified, the following four dealt almost exclusively with existential meaning achieved via different aspects of participants' professional work. 1) Professional interest and challenge: a sense of self-fulfillment, the diverse and dynamic nature of EM, and treating interesting 
Table 2. Participants' demographic characteristics

\begin{tabular}{lc}
\hline Variable & $\mathrm{n}(\%)$ \\
\hline Sex & \\
Male & $49(70)$ \\
Female & $21(30)$ \\
Age & \\
$<45$ & $23(32.8)$ \\
$45-60$ & $38(54.4)$ \\
$>60$ & $9(12.8)$ \\
Marital status & \\
Single & $6(8.6)$ \\
Divorced & $2(2.9)$ \\
Widowed & $1(1.4)$ \\
In a relationship (married or partnered) & $59(87.1)$ \\
Years of experience & \\
$<5$ & $16(22.9)$ \\
$5-25$ & $21(30)$ \\
$>25$ & $32(45.7)$ \\
Missing & $1(1.4)$ \\
\hline
\end{tabular}

Table 3. Physicians' burnout characteristics

\begin{tabular}{ll}
\hline Burnout indices variable & $\mathrm{n}(\%)$ \\
\hline Emotional exhaustion & \\
Low & $14(20)$ \\
Intermediate & $13(18.6)$ \\
High & $43(61.4)$ \\
Depersonalization & \\
Low & $23(32.9)$ \\
Intermediate & $11(15.7)$ \\
High & $36(51.4)$ \\
Personal achievement & \\
Low & $12(17.1)$ \\
Intermediate & $29(41.4)$ \\
High & $29(41.4)$ \\
Burned out (high on EE or DP) & $50(71.4)$ \\
\hline
\end{tabular}

cases. 2) Meaningful relationships: contact with people, satisfying team work, and relationships with patients and families. 3) Excitement and adrenaline rush: excitement and immediate satisfaction in the treatment of urgent cases, and the need for rapid response. 4) Providing meaningful assistance: providing correct diagnoses, exerting a positive impact on others, contributing to society, and saving lives. Only one cluster of responses, those concerning financial remuneration, addressed factors that were not directly related to meaning in different aspects of work.

Analysis of participants' identification of the aspects of their work that did not satisfy them revealed that the majority of the clusters (three) included stress- or worry-related factors. 1) Stress and worry related to relationships with other staff members: stressful interactions with specific staff members, a lack of "a conversation culture" among staff, and unprofessional staff members. 2) Stress and worry related to contact with patients and their fami-
Table 4. Results of Mann-Whitney tests performed to identify differences between burnout groups (burned out, not burned out) according to test variables

\begin{tabular}{ll}
\hline Variable & P-value \\
\hline Age & 0.17 \\
No. of children & 0.75 \\
Physical activity & 0.28 \\
Smoking & 0.05 \\
Alcohol consumption & 0.15 \\
Years of practicing medicine & 0.48 \\
Years of practicing emergency medicine & 0.12 \\
Clinical hours in emergency unit (per week) & 0.57 \\
Clinical hours outside the emergency unit (per week) & 0.06 \\
On-call days (per month) & 0.55 \\
Hours teaching students (per week) & 0.9 \\
Hours spent on research (per week) & 0.74 \\
Hours spent on administration (per week) & 0.09 \\
Weeks of vacation (per year) & 0.77 \\
Daily commute to work (in minute) & 0.07 \\
Job gives me meaning & 0.05 \\
Social support & 0.05 \\
Work-life balance & 0.05 \\
Feeling depressed & 0.001 \\
Lack of interest in daily activities & 0.05 \\
Feeling anxious & 0.001 \\
Feeling worried & 0.001 \\
Thoughts that I cannot get out of my head & 0.01 \\
Satisfaction with work & 0.001 \\
\hline
\end{tabular}

lies: overbearing patients and families, verbal and physical violence perpetrated by patients, and self-centered and impolite patients and family members. 3) Stress related to the dysfunctional elements of the medical system: difficulty transferring patients to relevant wards, ineffective procedures and protocols, suboptimal environmental conditions, administrative pressures, and having to treat patients with routine medical complaints.

Other clusters included work overload and insufficient pay. Only one additional cluster was related to the meaning that work gave to life. This cluster consisted of insufficient professional challenge and interest: lack of opportunities and time to engage in research and attend professional conferences.

The following clusters appeared in response to the question concerning which element participants would change to increase their satisfaction with work. 1) Reduce work overload: reduce workload (increase the size of the workforce and treat fewer patients) and decrease working time (decrease the number of hours and shifts worked, limit the duration of shifts to 8 hours, and increase vacation time). 2) Increase pay. 3) Improve work atmosphere: improve teamwork in the ED and enhance cooperation between different hospital units. 4) Increase professional autonomy. 


\section{DISCUSSION}

The aim of the current study was to determine burnout rates in full-time EPs in Israel and identify the variables that explain these rates, both positively and negatively. In addition, we examined the sense of meaning, which is a crucial variable that has received no attention in the literature on burnout in EPs.

Significant burnout was reported by $71 \%$ of respondents. These rates are significantly higher relative to those observed for physicians in other specialties (up to 39\%) ${ }^{7}$ and EPs in China (25.4\%) ${ }^{11}$ but similar to those observed for EPs in the US (60\% to 65\%). ${ }^{12}$

Interestingly, high burnout levels were observed in participants who reported strong feelings of competency and work satisfaction, and $82 \%$ of participants reported medium or high competency levels (personal accomplishment), which was a similar finding to those of previous studies. ${ }^{15}$

The main variables that were associated with burnout were work-life balance, satisfaction with work, a sense of existential meaning derived from work, social support, depressive symptoms, worry, stress, and preoccupying thoughts. Some of these variables correspond to the findings of previous studies. For example, worklife balance has repeatedly been found to predict burnout in internal medicine physicians, surgeons, and residents. ${ }^{23-26}$ Israel has a strong pronatalist culture, which stresses the significance of family life; accordingly, work-life balance was a significant factor in decreasing burnout levels, which was also reported in another Israeli study. ${ }^{27}$ Satisfaction with work was a protective factor against burnout, and distress and worry were associated with increased burnout. ${ }^{26,28,29}$ As in other studies, there was no significant relationship observed between participants' demographic characteristics and burnout levels. ${ }^{12,13}$

Two other factors significantly predicted burnout: worry, which was also associated with burnout in previous studies, ${ }_{1}^{29}$ and meaning derived from work. While previous studies reported that a sense of competence and work satisfaction predicted burnout, to the best of our knowledge, no studies have identified the existential meaning derived from work as a significant protective factor against burnout in physicians. Individuals may feel professionally competent and satisfied with their work without necessarily achieving personal fulfillment or a sense of meaning in life.

The psychoanalytic-existential approach assumes that the root of career burnout lies in the human need to believe that our lives are meaningful and what we do-and consequently, who we areare important and significant. ${ }^{30}$ Frank ${ }^{16}{ }^{16}$ suggested that the striving to find meaning in one's life is the primary motivational force in man. Becker ${ }^{31}$ posited that work is a frequently chosen path to existential significance for many people.
The assumption that people experience burnout when they feel that their work is no longer significant has been supported in the past. ${ }^{32}$ Nevertheless, some studies have reported that medical students emphasized factors other than a sense of meaning as important in choosing a career in EM. These factors include diversity in clinical pathology, emphasis on acute care, previous employment in an emergency setting, and flexibility in both practice location and work schedule in final-year medical students, ${ }^{33}$ and hospital orientation, medical lifestyle, and varied scope of practice in entry-level medical students. ${ }^{34}$

In contrast to these findings, the results of the current study suggest that a sense of meaning was an important influential factor in work satisfaction and burnout in the later stages of EPs' careers. In the early stages of professional training, it may be difficult to envisage the ways in which one's future medical practice should be developed to maximize individual happiness. Although less emphasis is placed on the aspects of EPs work concerning a sense of meaning in the early stages of their careers, a shift in focus is expected in later stages, as they may be exposed to unanticipated factors. For instance, while some research has suggested that entry-level medical students are less likely to report that social orientation was an important factor in their interest in $\mathrm{EM}_{1}^{34}$ the results of the current study show a shift in emphasis toward meaningful relationships and the provision of meaningful assistance in the later stages of EPs' careers. This shift in emphasis may contribute to the high levels of burnout observed in fulltime EPs in this and previous studies.

Considering the high price of burnout, there is clearly a need to develop interventions to counter burnout. In addition to factors that have previously been associated with burnout, the current findings highlighted the importance of existential meaning as a protective factor against burnout. This finding could guide the development of specific intervention programs that focus on methods of increasing the sense of meaning that physicians derive from their work. This is important, as burnout intervention programs often focus primarily on the objective aspects of work (e.g., workload or payment).

In view of the centrality of the meaning derived from work, it is surprising that so few studies have examined the processes involved in enhancing meaningfulness at work. ${ }^{18}$ Furthermore, despite serious consequences, few studies have assessed interventions designed to reduce burnout levels and enhance the meaning derived from work. ${ }^{22}$ These studies were characterized by small sample sizes and focused mainly on individual, rather than organizational interventions. Nevertheless, there is growing evidence that interventions designed to enhance the meaning derived from work decrease the incidence of burnout in physicians. ${ }^{35,36}$ For in- 
stance, physicians' ability to focus on the most meaningful aspects of their work is strongly negatively associated with burnout. ${ }^{35}$ Mindfulness-based interventions have been associated with significant improvements in burnout scores and mental well-being in a broad range of healthcare providers. ${ }^{37}$

The analysis of responses to the open-ended questions could offer preliminary directions for future research, such as examination of the centrality of meaning derived from the emotional aspects of medicine (e.g., forging relationships with other staff members and patients and their families). Further exploration of the involvement of the process of enhancing existential meaningfulness in EPs necessitates in-depth interviews that could facilitate the examination of different aspects of physicians' sense of existential meaning and how it develops over time and in different career stages. Specifically, identifying meaningfulness enhancing tactics may allow those tactics to be taught to burned-out physicians, decreasing their burnout levels.

The strengths of the present study include the broad and varied sample recruited from several medium and large hospitals, which increased the generalizability of the results. ${ }^{4}$ The study's limitations include the sample size and response rate and the intentionally limited breadth of the questions.

\section{CONFLICT OF INTEREST}

No potential conflict of interest relevant to this article was reported.

\section{REFERENCES}

1. Shirom A, Melamed S, Toker S, Berliner S, Shapira I. Burnout and health review: current knowledge and future research directions. In: Hodgkinson GP, Ford JK, editors. International review of industrial and organizational psychology. New York, NY: Wiley; 2005. p.269-308.

2. Maslach $C$, Jackson SE, Leiter MP. Maslach burnout inventory. Palo Alto, CA: Consulting Psychologists Press; 1996.

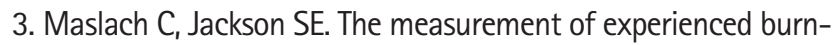
out. J Occup Behav 1981;2:99-113.

4. Arora $M$, Asha S, Chinnappa J, Diwan AD. Review article: burnout in emergency medicine physicians. Emerg Med Australas 2013;25:491-5.

5. Friedman IA. Burnout in teachers: the concept and its unique core meaning. Educ Psychol Meas 1993;53:1035-44.

6. Ramirez AJ, Graham J, Richards MA, Cull A, Gregory WM. Mental health of hospital consultants: the effects of stress and satisfaction at work. Lancet 1996;347:724-8.
7. Shanafelt TD, Boone $S$, Tan $L$, et al. Burnout and satisfaction with work-life balance among US physicians relative to the general US population. Arch Intern Med 2012;172:1377-85.

8. Shanafelt TD, Sloan JA, Habermann TM. The well-being of physicians. Am J Med 2003;114:513-9.

9. Meier DE, Back AL, Morrison RS. The inner life of physicians and care of the seriously ill. JAMA 2001;286:3007-14.

10. Einav $S$, Shalev AY, Ofek $H$, Freedman $S$, Matot I, Weiniger CF. Differences in psychological effects in hospital doctors with and without post-traumatic stress disorder. Br J Psychiatry 2008;193:165-6.

11. Xiao Y, Wang J, Chen S, et al. Psychological distress, burnout level and job satisfaction in emergency medicine: a cross-sectional study of physicians in China. Emerg Med Australas 2014; 26:538-42.

12. Goldberg R, Boss RW, Chan L, et al. Burnout and its correlates in emergency physicians: four years' experience with a wellness booth. Acad Emerg Med 1996;3:1156-64.

13. Kuhn G, Goldberg R, Compton S. Tolerance for uncertainty, burnout, and satisfaction with the career of emergency medicine. Ann Emerg Med 2009;54:106-13.e6.

14. Estryn-Behar M, Doppia MA, Guetarni K, et al. Emergency physicians accumulate more stress factors than other physicians-results from the French SESMAT study. Emerg Med J 2011;28:397-410.

15. Lloyd S, Streiner D, Shannon S. Burnout, depression, life and job satisfaction among Canadian emergency physicians. J Emerg Med 1994;12:559-65.

16. FrankI VE. Man's search for meaning. Boston, MA: Beacon Press; 1985.

17. Langle A. Burnout: existential meaning and possibilities of prevention. Eur Psychother 2003;4:129-43.

18. Vuori T, San E, Kira M. Meaningfulness-making at work. Qual Res Organ Manag Int J 2012;7:231-48.

19. Loonstra B, Brouwers A, Tomic W. Feelings of existential fulfilment and burnout among secondary school teachers. Teach Teach Educ 2009;25:752-7.

20. Tomic $W$, Tomic E. Existential fulfillment and burnout among principals and teachers. J Beliefs Values 2008;29:11-27.

21. Kushnir T, Levhar $\mathrm{C}$, Cohen AH. Are burnout levels increasing? The experience of Israeli primary care physicians. Isr Med Assoc J 2004;6:451-5.

22. Shanafelt TD, Balch CM, Bechamps GJ, et al. Burnout and career satisfaction among American surgeons. Ann Surg 2009; 250:463-71.

23. Linzer $M$, Visser MR, Oort FJ, et al. Predicting and preventing physician burnout: results from the United States and the 
Netherlands. Am J Med 2001;111:170-5.

24. Dyrbye LN, Shanafelt TD, Balch CM, Satele D, Sloan J, Freischlag J. Relationship between work-home conflicts and burnout among American surgeons: a comparison by sex. Arch Surg 2011;146:211-7.

25. Geurts S, Rutte C, Peeters M. Antecedents and consequences of work-home interference among medical residents. Soc Sci Med 1999;48:1135-48.

26. Visser MR, Smets EM, Oort FJ, De Haes HC. Stress, satisfaction and burnout among Dutch medical specialists. CMAJ 2003; 168:271-5.

27. Barilan A, Haimov T, Doplet $K$, Matnick S, Vinker S, Kitai E. Workload, burnout and need to recover among female residents in internal medicine and family medicine. Harefuah $2011 ; 150$ : 625-9, 690.

28. Rath K, Huffman L, Carpenter K, Fowler J. Burnout is associated with decreased career satisfaction and psychosocial distress among members of the Society of Gynecologic Oncology (SGO). In: Society of Gynecologic Oncology. 45th Annual Meeting on Women's Cancer; 2014 Mar 22-25; Tampa, USA. Chicago, IL: Society of Gynecologic Oncology; 2014.

29. Richardson DR, Hammock GS, Smith SM, Gardner W, Signo M. Empathy as a cognitive inhibitor of interpersonal aggression. Aggress Behav 1994;20:275-89.
30. Pines AM. Burnout: an existential perspective. In: Schaufeli $W$, Maslach C, Marek T, editors. Professional burnout: recent developments in theory and research. Washington, DC: Taylor and Francis; 1993. p.33-52.

31. Becker E. The denial of death. London: Souvenir Press; 2007.

32. Etzion D, Pines A. Sex and culture in burnout and coping among human service professionals: a social psychological perspective. J Cross-Cult Psychol 1986;17:191-209.

33. Kazzi AA, Langdorf MI, Ghadishah D, Handly N. Motivations for a career in emergency medicine: a profile of the 1996 US applicant pool. CJEM 2001;3:99-104.

34. Scott IM, Abu-Laban RB, Gowans MC, Wright BJ, Brenneis FR. Emergency medicine as a career choice: a descriptive study of Canadian medical students. CJEM 2009;11:196-206.

35. Shanafelt TD. Enhancing meaning in work: a prescription for preventing physician burnout and promoting patient-centered care. JAMA 2009;302:1338-40.

36. Krasner MS, Epstein RM, Beckman $H$, et al. Association of an educational program in mindful communication with burnout, empathy, and attitudes among primary care physicians. JAMA 2009;302:1284-93.

37. Goodman MJ, Schorling JB. A mindfulness course decreases burnout and improves well-being among healthcare providers. Int J Psychiatry Med 2012;43:119-28. 
Supplementary Table 1. Results concerning the other variables included in the survey

\begin{tabular}{|c|c|}
\hline Variable & n (\%) \\
\hline \multicolumn{2}{|l|}{ No. of children } \\
\hline 0 & $9(12.9)$ \\
\hline 1 & $7(10)$ \\
\hline 2 & $27(38.6)$ \\
\hline 3 & $11(15.7)$ \\
\hline 4 & $13(18.6)$ \\
\hline 5 & $2(2.9)$ \\
\hline 6 & $0(0)$ \\
\hline 7 & $1(1.4)$ \\
\hline \multicolumn{2}{|l|}{ Physical activity } \\
\hline Never & $11(15.7)$ \\
\hline Rarely & $26(37.1)$ \\
\hline Regularly & $21(30)$ \\
\hline Engage in a lot of physical activity & $9(12.9)$ \\
\hline Missing & $3(4.3)$ \\
\hline \multicolumn{2}{|l|}{ Smoking } \\
\hline Never & $51(72.9)$ \\
\hline Rarely & $12(17.1)$ \\
\hline Medium degree & $7(10)$ \\
\hline Heavy smoker & $0(0)$ \\
\hline \multicolumn{2}{|l|}{ Alcohol } \\
\hline Never & $23(32.9)$ \\
\hline Rarely & $37(52.9)$ \\
\hline Medium degree & $10(14.3)$ \\
\hline Heavy drinking & $0(0)$ \\
\hline \multicolumn{2}{|l|}{ Academic rank } \\
\hline Instructor & $13(18.6)$ \\
\hline Assistant professor & $5(7.1)$ \\
\hline Associate professor & $2(2.9)$ \\
\hline Full Professor & $3(4.3)$ \\
\hline Emeritus & $0(0)$ \\
\hline Not applicable & $47(67.1)$ \\
\hline \multicolumn{2}{|l|}{ Years of practicing medicine } \\
\hline$<5$ & $10(14.3)$ \\
\hline $5-10$ & $6(8.6)$ \\
\hline $11-15$ & $8(11.4)$ \\
\hline $16-20$ & $6(8.6)$ \\
\hline $21-25$ & $7(10)$ \\
\hline$>25$ & $32(45.7)$ \\
\hline \multicolumn{2}{|l|}{ Years of practicing emergency medicine } \\
\hline$<5$ & $23(32.9)$ \\
\hline $5-10$ & $16(22.9)$ \\
\hline $11-15$ & $10(14.3)$ \\
\hline $16-20$ & $10(14.3)$ \\
\hline $21-25$ & $3(4.3)$ \\
\hline$>25$ & $6(8.6)$ \\
\hline Missing & $2(2.9)$ \\
\hline \multicolumn{2}{|l|}{ Other formal specialty training } \\
\hline None & $15(21.4)$ \\
\hline Internal medicine & $36(51.4)$ \\
\hline Other & $19(27.2$ \\
\hline \multicolumn{2}{|l|}{ Subspecialty training } \\
\hline None & $45(64.3)$ \\
\hline Emergency medicine & $18(25.7)$ \\
\hline Other & $7(10)$ \\
\hline
\end{tabular}

(Continuing)
Supplementary Table 1. Continued

\begin{tabular}{|c|c|}
\hline Variable & n (\%) \\
\hline \multicolumn{2}{|c|}{ Clinical hours in emergency unit (per week) } \\
\hline$<30$ & $18(25.7)$ \\
\hline $30-40$ & $22(31.4)$ \\
\hline $41-50$ & $25(35.7)$ \\
\hline $51-60$ & $4(5.7)$ \\
\hline Missing & $1(1.4)$ \\
\hline \multicolumn{2}{|c|}{ Clinical hours outside the emergency unit (per week) } \\
\hline None & $15(21.4)$ \\
\hline$<30$ & 45 (64.3) \\
\hline $30-40$ & $7(10)$ \\
\hline $41-50$ & $2(2.9)$ \\
\hline $51-60$ & $0(0)$ \\
\hline$>70$ & $1(1.4)$ \\
\hline \multicolumn{2}{|c|}{ On-call days (per month) } \\
\hline 0 & $3(4.3)$ \\
\hline $1-2$ & $27(38.6)$ \\
\hline $3-5$ & $18(25.7)$ \\
\hline $6-10$ & $13(18.6)$ \\
\hline$>10$ & $9(12.9)$ \\
\hline \multicolumn{2}{|c|}{ Hours teaching students (per week) } \\
\hline 0 & $22(31.4)$ \\
\hline $1-4$ & $22(31.4)$ \\
\hline $5-10$ & $9(12.9)$ \\
\hline $11-15$ & $11(15.7)$ \\
\hline $16-20$ & $5(7.1)$ \\
\hline$<20$ & $1(1.4)$ \\
\hline \multicolumn{2}{|c|}{ Hours spent on research (per week) } \\
\hline 0 & $19(27.1)$ \\
\hline $1-4$ & $41(58.6)$ \\
\hline $5-10$ & $7(10)$ \\
\hline $11-15$ & $2(2.9)$ \\
\hline $16-20$ & $0(0)$ \\
\hline$<20$ & $0(0)$ \\
\hline Missing & $1(1.4)$ \\
\hline \multicolumn{2}{|c|}{ Hours spent on administration (per week) } \\
\hline 0 & $14(20)$ \\
\hline $1-4$ & $39(55.7)$ \\
\hline $5-10$ & $5(7.1)$ \\
\hline $11-15$ & $5(7.1)$ \\
\hline $16-20$ & $4(5.7)$ \\
\hline$<20$ & $3(4.3)$ \\
\hline \multicolumn{2}{|c|}{ Weeks of vacation (per year) } \\
\hline$<1$ & $4(5.7)$ \\
\hline $1-2$ & $35(50)$ \\
\hline $3-4$ & $27(38.6)$ \\
\hline$>4$ & $4(5.7)$ \\
\hline \multicolumn{2}{|c|}{ Daily commute to work (in minute) } \\
\hline$<10$ & $7(10)$ \\
\hline $10-20$ & $12(17.1)$ \\
\hline $21-30$ & 15 (21.4) \\
\hline$>30$ & $36(51.4)$ \\
\hline \multicolumn{2}{|c|}{ Malpractice lawsuit (in the last 5 years) } \\
\hline 0 & 68 (97.1) \\
\hline $1-5$ & $2(2.9)$ \\
\hline $6-10$ & $0(0)$ \\
\hline$>10$ & $0(0)$ \\
\hline
\end{tabular}

